\title{
Simulation of Lag and Current Slump in AlGaN/GaN HEMTs as Affected by Buffer Trapping
}

\author{
A. Nakajima, K. Itagaki and K. Horio \\ Shibaura Institute of Technology, 307 Fukasaku, Saitama 337-8570, Japan \\ horio@sic.shibaura-it.ac.jp
}

\begin{abstract}
Two-dimensional transient simulations of AlGaN/GaN HEMTs are performed in which a deep donor and a deep acceptor are considered in a semi-insulating buffer layer. It is shown that lag phenomena and current slump could be reproduced. Particularly, it is shown that gate lag is correlated with relatively high source access resistance of $\mathrm{AlGaN} / \mathrm{GaN}$ HEMTs, and that drain lag could be a major cause of current slump. The current slump is more pronounced when the deep-acceptor density in the buffer layer is higher and when an off-state drain voltage is higher, because trapping effects become more significant. It is concluded that an acceptor density in the buffer layer should be made low to minimize current slump, although current cutoff behavior may be degraded when the gate length is short.
\end{abstract}

\section{Introduction}

Recently, AlGaN/GaN HEMTs have received great attention because of their potential applications to high power microwave devices [1]. However, slow current transients are often observed even if the drain voltage $V_{\mathrm{D}}$ or gate voltage $V_{\mathrm{G}}$ is changed abruptly (called drain lag or gate lag) [2]. The slow transients mean that dc $I$ $V$ curves and RF $I-V$ curves become quite different, resulting in lower RF power available than that expected from dc operation [1,2]. This is called power slump or current slump. These are serious problems, and there are many experimental works reported [1-5]. However, only a few theoretical works are made recently[4,6], where effects of a donor-type surface state (near the valence band) or effects of a bulk deep acceptor $(\sim 1 \mathrm{eV}$ above the midgap of $\mathrm{GaN})$ are studied for gate lag or pulsed $I-V$ curves in AlGaN/GaN HEMTs. But, the type of traps and their energy levels seemed to be artificial. So, in this work, we have made simulations of AlGaN/GaN HEMTs with a semi-insulating buffer layer in which trap levels based on experiments are considered, as in our previous work on GaN MESFETs [7], and showed that the lag phenomena and current slump could be reproduced. Particularly, we show that the gate lag is correlated with relatively high source access resistance of $\mathrm{AlGaN} / \mathrm{GaN}$ HEMTs, and also give a way to reduce the current slump.

\section{Physical Model}

Fig.1 shows an analyzed AlGaN/GaN HEMT structure. As a model for the semiinsulating buffer layer, we use a three-level compensation model which includes a 


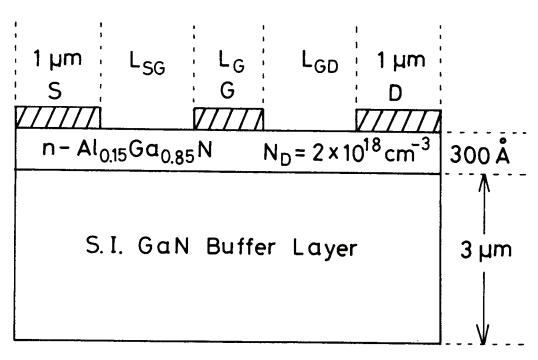

Figure 1: Modeled AlGaN/GaN HEMT analyzed in this study.

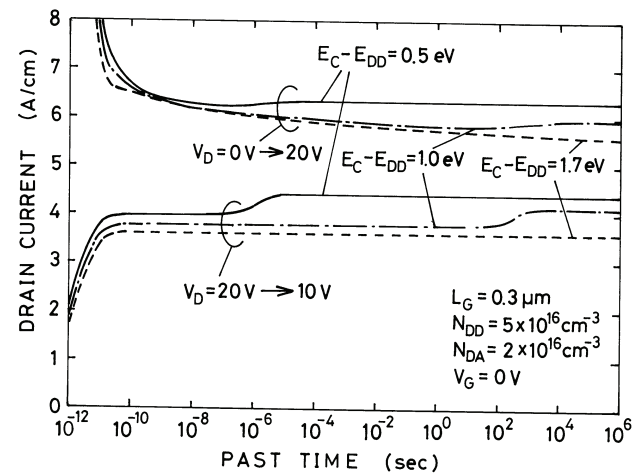

Figure 2: Calculated drain-current responses as a parameter of $E_{\mathrm{DD}}$ when $V_{\mathrm{D}}$ is raised abruptly from $0 \mathrm{~V}$ to $20 \mathrm{~V}$ (upper) or when $V_{\mathrm{D}}$ is lowered abruptly from $20 \mathrm{~V}$ to $10 \mathrm{~V}$ (lower). $V_{\mathrm{G}}=0 \mathrm{~V}$.

shallow donor, a deep donor and a deep acceptor. Some experiments show that two levels $\left(E_{\mathrm{C}}-1.7 \mathrm{eV}, E_{\mathrm{C}}-2.85 \mathrm{eV}\right)$ are associated with current slump in GaN-based FETs [2], so that we use energy levels of $E_{\mathrm{C}}-2.85 \mathrm{eV}$ (or $E_{\mathrm{V}}+0.6 \mathrm{eV}$ ) for the deep acceptor and of $E_{\mathrm{C}}-1.7 \mathrm{eV}$ for the deep donor. Other experiments show shallower energy levels for deep donors in $\mathrm{GaN}$ [8], and hence we vary the deep donor's energy level $E_{\mathrm{DD}}$ as a parameter. Basic equations are Poisson's equation including ionized deep-level terms, continuity equations for electrons and holes which include carrier loss rates via the deep levels, and rate equations for the deep levels. We have calculated the drain current responses when $V_{\mathrm{D}}$ and/or $V_{\mathrm{G}}$ are changed abruptly.

\section{Lag Phenomena}

Fig.2 shows calculated responses of drain current $I_{\mathrm{D}}$ when $V_{\mathrm{D}}$ is raised or lowered abruptly, where $V_{\mathrm{G}}$ is kept constant. When $V_{\mathrm{D}}$ is raised, $I_{\mathrm{D}}$ overshoots the steady-state value, because electrons are injected into the buffer layer, and deep traps there need certain time to capture these electrons. On the other hand, when $V_{\mathrm{D}}$ is lowered, $I_{\mathrm{D}}$ remains at a low value for some periods and begins to increase slowly, showing drain lag behavior. It is understood that the drain currents begin to increase as the deep donors begin to emit electrons. These drain lags are also reported experimentally in AlGaN/GaN HEMTs [2,5].

We have next calculated a case when $V_{\mathrm{G}}$ is also changed. Fig.3 shows calculated turnon characteristics when $V_{\mathrm{G}}$ is changed from the threshold voltage $V_{\text {th }}$ to $0 \mathrm{~V}$, with onstate drain voltage $V_{\text {Don }}$ as a parameter. The characteristics are similar to those in Fig.2, and hence the change of $V_{\mathrm{D}}$ (drain lag) is regarded as essential in this case. However, as seen in the uppermost curve of Fig.3, some transients are observed when only $V_{\mathrm{G}}$ is changed. This indicates that gate lag occurs due to deep levels in the buffer layer. We will describe below why the gate lag arises. Fig.4 shows a comparison of (a) conduction-band-edge energy profiles, (b) electron density profiles, and (c) ionized-deep donor density $N_{\mathrm{DD}}{ }^{+}$profiles between the off state and the on state. Note that only $V_{\mathrm{G}}$ is different here. From Fig.4(a), in the on state, some potential drops are 


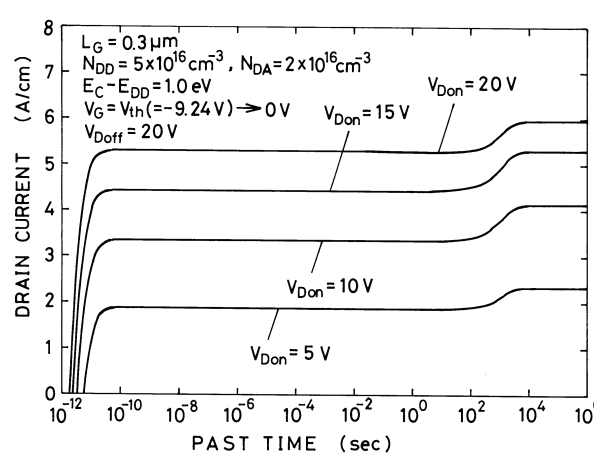

Figure 3: Calculated turn-on characteristics when $V_{\mathrm{G}}$ is changed from threshold voltage $V_{\text {th }}$ to $0 \mathrm{~V}$, with on-state drain voltage $V_{\text {Don }}$ as a parameter. Off-state drain voltage $V_{\text {Doff }}$ $=20 \mathrm{~V} \cdot E_{\mathrm{C}}-E_{\mathrm{DD}}=1.0 \mathrm{eV}$.

Figure 4: (a) Conduction-band-edge energy profiles, (b) electron density profiles, and (c) ionized deep-donor density $N_{\mathrm{DD}}{ }^{+}$profiles when only $V_{\mathrm{G}}$ is different. The left is for $V_{\mathrm{G}}=$ $V_{\text {th }}=-9.24 \mathrm{~V}$ and $V_{\mathrm{D}}=20 \mathrm{~V}(\mathrm{OFF})$, and the right is for $V_{\mathrm{G}}=0 \mathrm{~V}$ and $V_{\mathrm{D}}=20 \mathrm{~V}(\mathrm{ON})$.
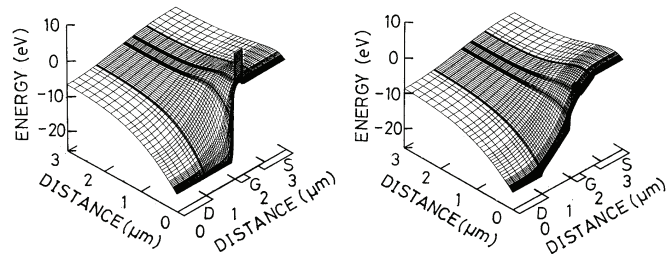

(a)
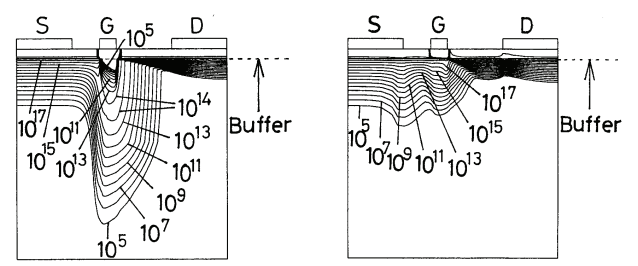

(b)

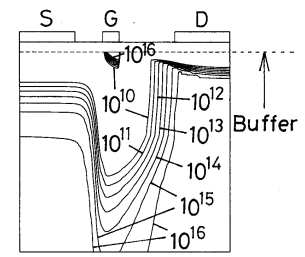

OFF

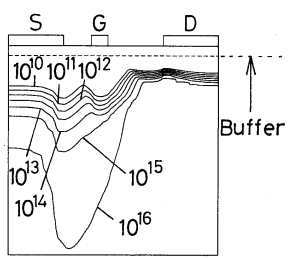

ON

(c)

observed between source and gate, indicating that source access resistance becomes important. It is understood that due to this potential drop at the source side, when $V_{\mathrm{G}}$ becomes negative and the channel is depleted, electrons do not all flow into the source and drain electrodes, but can be injected into the buffer layer as seen in Fig.4(b). These electrons are captured by deep donors, and hence $N_{\mathrm{DD}}{ }^{+}$decreases in the off state, as seen in Fig.4(c). Because of this increase in negative space charges in the buffer layer, even if $V_{\mathrm{G}}$ is switched on, $I_{\mathrm{D}}$ remains at a low value until the deep donors begin to emit electrons, showing gate-lag behavior.

\section{Current Slump}

Next, we have studied dependence of current slump on deep-level densities in the buffer layer. Fig. 5 shows calculated $I_{\mathrm{D}}-V_{\mathrm{D}}$ curves for different deep-acceptor density $N_{\mathrm{DA}}$. In this figure, we plot by point (x) the drain current at $t=10^{-8} \mathrm{~s}$ after $V_{\mathrm{G}}$ is switched on. This is obtained from the turn-on characteristics, and this curve corresponds to a quasi-pulsed $I-V$ curve with pulse width of $10^{-8} \mathrm{~s}$. It is seen the drain currents in the pulsed $I-V$ curves become rather lower than those in the steady state, showing current slump behavior. This type of current reduction is commonly observed experimentally in AlGaN/GaN HEMTs. It is clearly seen that the current slump is more pronounced for higher $N_{\mathrm{DA}}$. This is because trapping effects become more remarkable due to higher ionized (empty) deep-donor density $N_{\mathrm{DD}}{ }^{+}$. It is concluded that the acceptor density must be made low to minimize current slump. 


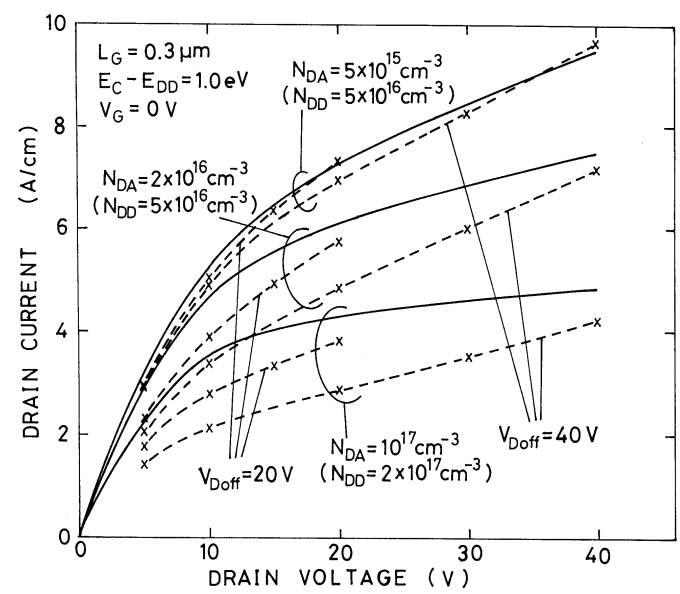

Figure 5: Steady-state $I-V$ curves $\left(V_{\mathrm{G}}=0 \mathrm{~V}\right.$; solid lines) and quasi-pulsed $I-V$ curves $(\mathrm{x} ; t=$ $10^{-8} \mathrm{~s}$ ) from off points for AlGaN/GaN HEMTs with different $N_{\mathrm{DA}} . E_{\mathrm{C}}-E_{\mathrm{DD}}=1.0 \mathrm{eV}$.

Finally, we have studied dependence of current slump on an off-state drain voltage $V_{\text {Doff. }}$ As seen in Fig.5, the current slump is more pronounced for higher $V_{\text {Doff, }}$ and contribution of drain lag to it becomes large. This is because for higher $V_{\mathrm{D}}$, electrons are injected deeper into the buffer layer and more electrons are captured by the deep donors, resulting in heavier current reduction. This tendency is also reported experimentally in AlGaN/GaN HEMTs [3].

\section{Conclusion}

Two-dimensional transient simulations of AlGaN/GaN HEMTs have been made in which a three-level compensation model is adopted for the buffer layer. The lag phenomena and current slump could be reproduced. Particularly, it has been shown that the gate lag is correlated with relatively high source access resistance of AlGaN/GaN HEMTs, and that the drain lag could be a major cause of current slump. It is concluded that an acceptor density in the buffer layer should be made low to minimize current slump, although current cutoff behavior may be degraded when the gate length is short.

\section{References}

[1] U. K. Mishra, P. P. Parikh and Y.-F. Wu, Proc. IEEE, vol.90, pp.1022-1031, 2002.

[2] S. C. Binari, P. B. Klein and T. E. Kazior, Proc. IEEE, vol.90, pp.1048-1058, 2002.

[3] A. Koudymov et al., IEEE Electron Device Lett., vol.24, pp.680-682, 2003.

[4] G. Meneghesso et al., IEEE Trans. Electron Devices, vol.51, pp.1554-1561, 2004.

[5] V. Desmaris et al, IEEE Trans. Electron Devices, vol.53, pp.2413-2417, 2006.

[6] N. Braga et al., Proc. CSIC Symp., pp.287-290, 2004.

[7] K. Horio, K. Yonemoto, H. Takayanagi and H. Nakano, J. Appl. Phys., vol.98, no.12, pp.124502 1-7, 2005.

[8] H. Morkoc, Nitride Semiconductors and Devices, Springer-Verlag, 1999. 